\title{
Acupressure therapy for insomnia in adolescents: a polysomnographic study
}

\author{
This article was published in the following Dove Press journal: \\ Neuropsychiatric Disease and Treatment \\ 24 January 2013 \\ Number of times this article has been viewed
}

\author{
Marco Carotenuto' \\ Beatrice Gallai ${ }^{2}$ \\ Lucia Parisi ${ }^{3}$ \\ Michele Roccella ${ }^{3}$ \\ Maria Esposito' \\ 'Sleep Clinic for Developmental \\ Age, Clinic of Child and Adolescent \\ Neuropsychiatry, Second University \\ of Naples, Naples, ${ }^{2}$ Unit of Child \\ and Adolescent Neuropsychiatry, \\ University of Perugia, Perugia, ${ }^{3}$ Child \\ Neuropsychiatry, Department of \\ Psychology, University of Palermo, \\ Palermo, Italy
}

Background: The purpose of this study was to assess the efficacy of acupressure therapy in a sample of adolescents with insomnia using a standard polysomnographic evaluation.

Methods: For this study, 25 adolescents affected by psychophysiological insomnia (mean age $15.04 \pm 1.18$ years, 12 boys) were enrolled. A device known as the Sea-Band ${ }^{\circledR}$ was used by the patients in order to improve their symptoms related to difficulty in falling asleep. All subjects enrolled underwent two sets of consecutive overnight polysomnographic studies in the Sleep Laboratory of the Clinic of Child and Adolescent Neuropsychiatry, comprising two studies at baseline (before treatment) and another two studies at the end of 6 months of treatment.

Results: At the end of 6 months of treatment, there was a significant increase in all macrostructural parameters of sleep duration, and a reduction in sleep onset latency, wake after sleep onset, and stage 2 sleep. Moreover, the study group showed a significant increase in percent sleep efficiency $(P<0.001)$ and in slow wave sleep representation.

Conclusion: Acupressure is a noninvasive, safe, and effective method for the management of insomnia in adolescents, with good compliance and no adverse effects.

Keywords: insomnia, adolescence, acupressure, polysomnography

\section{Introduction}

In clinical practice, sleep disturbances are a frequent reason for pediatric consultation, ${ }^{1}$ and may be organic in nature (eg, obstructive sleep apnea) or behavioral (ie, limit setting disorder, sleep onset association, insomnia). ${ }^{2}$ However, when unrecognized, the problem can become chronic and increase the risk of behavioral ${ }^{3}$ and/or organic abnormalities. ${ }^{4,5}$ In fact, the presence of sleep disturbances during infancy may be considered as predictive of subsequent emotional or behavioral problems during adolescence. ${ }^{6,7}$ In general, insomnia seems to be one of the most common sleep complaints, with a prevalence of $40 \%$ in adulthood, ${ }^{8}$ and about $40 \%$ of adolescents report some form of sleep problem, including issues of sleep apnea, snoring, and sleep difficulty. ${ }^{9-11}$

Compared with prepubertal youth, adolescents seem to need more sleep, spend less time in slow wave sleep and latency to rapid-eye-movement (REM) sleep, and have a propensity for delayed sleep phase syndrome. In addition, social factors, including early school start times, after-school employment, and extracurricular activities contribute to limited sleep and disrupted sleep patterns, making adolescents vulnerable to excessive sleepiness. Poor sleep adversely affects cognitive function, academic performance, and attentive ability, ${ }^{12-14}$ and is associated with poor emotional and physical health ${ }^{3,15}$ conduct problems, and substance use. ${ }^{16-19}$
Correspondence: Maria Esposito

Clinic of Child and Adolescent

Neuropsychiatry, Second University of Naples, Via Sergio Pansini 5 PAD XI 80 I 3 I Naples, Italy

Tel +39815666988

Fax +398 I566 6694

Email maria.esposito2@unina2.it 
Moreover, adolescents tend to report insomnia as the most frequent sleep disorder, described as disorders of initiating and maintaining sleep, restless sleep, resistance at bedtime, cosleeping, alterations of sleep hygiene, and early awakening in the morning associated with headache. ${ }^{20}$

Insomnia can be treated with medication, herbal therapy, and psychologic or physical therapy. Commonly used medications include hypnotic/sedative drugs, but may have adverse effects, including impairment of memory, drug resistance, dependency, and addiction. ${ }^{21}$ Among the nondrug therapies for insomnia, acupressure, a method used for over 5000 years in Eastern medicine, is becoming increasingly popular worldwide.

According to Eastern medicine, there is an equilibrium between the bipolar forces of Yin and Yang, and disease can result from disruption of such equilibrium. Acupuncture and acupressure are believed to restore equilibrium. Acupressure involves using the fingers, thumbs, palms, heels of the hand, and elbows to apply pressure and stimulate specific points along the meridians (or energy channels) of the body. Acupressure is rapidly gaining acceptance as a safe, cost-effective, noninvasive, and nonpharmacological form of therapy. ${ }^{22}$ Use of acupressure is based on stimulation of meridians, a network of energy pathways throughout the body, to increase the flow of so-called "qii" (bioenergy), subsequently altering the experience of symptoms. ${ }^{23}$ Acupressure is applied to specific points using the finger, hand, elbow, foot, and/or an acupressure Sea-Band ${ }^{\circledR}$ (SeaBand, Leicestershire, UK) which stimulates these pathways and increases the flow of qi. Studies have suggested the efficacy of acupressure therapy in sleep regulation ${ }^{24}$ and, in particular, acupressure at the HT-7 (Shen Men) point seems to have a therapeutic effect in patients with disorders of initiating and maintaining sleep. ${ }^{21}$

In the clinical literature, there are also several reports of the efficacy of acupressure therapy in various other conditions, including nocturnal enuresis, ${ }^{25}$ body weight and serum lipid levels, ${ }^{26}$ and migraine-associated nausea, ${ }^{27}$ which may be associated with sleep disorders. ${ }^{4,20,28}$ The aim of the present study was to assess the efficacy and safety of an HT-7 point acupressure system when used to treat insomnia in adolescents using a standard polysomnographic evaluation.

\section{Materials and methods}

\section{Study population}

Twenty-five adolescents affected by psychophysiological insomnia (12 boys, 13 girls, mean age $15.04 \pm 1.18$ years) were enrolled in this study. The Sea-Band device was given to the patients in order to improve their symptoms related to difficulty falling asleep. The Sea-Band is an elastic wristband with a $1 \mathrm{~cm}$ protruding round plastic button, and the device applies continual pressure to the HT-7 acupuncture point with the aim of decreasing or completely eliminating insomnia. The HT-7 point is located on the wrist, at the ulnar end of the transverse crease of the wrist, in the depression on the radial side of the flexor carpi ulnaris tendon. Sea-Bands were applied bilaterally at the Shen Men point on both wrists, starting from the usual bedtime $(10 \mathrm{pm})$ to the usual awakening time (7 am) every night for a period of 6 months.

All enrolled subjects underwent consecutive overnight polysomnographic studies in the Sleep Laboratory of the Clinic of Child and Adolescent Neuropsychiatry, comprising two at baseline (before treatment) and another two at the end of 6 months of treatment. The results obtained from the first night at baseline and at the end of 6 months of treatment were not included in the analysis to avoid the "first-night" effect. $^{29}$

All subjects were recruited from the same urban area, were of Caucasian origin, and of middle class socioeconomic status. An initial screening interview was carried out by a child and adolescent neuropsychiatrist. A preliminary diagnosis for inclusion in the study was made according to International Classification of Sleep Disorders (ICSD-2) criteria. The subjects had to report having at least two symptoms of insomnia (fragmented sleep, frequent awakenings, early morning awakenings followed by an inability to fall back to sleep, or feeling tired in the morning despite having spent a normal period of time in bed) for at least 2 years beforehand, which were not related to an obvious environmental stressor. Potential participants with any concurrent medical, psychological, or psychiatric factors which might account for their sleep difficulties were excluded. Other exclusion criteria were presence of other sleep disorders, history of alcohol or drug abuse, current treatment with psychoactive drugs, or concurrent psychotherapy.

All evaluations were performed after informed consent was obtained from the parents, according to the Declaration of Helsinki as revised in 2000. The study was approved by the departmental ethics committee of the Second University of Naples.

\section{Polysomnographic sleep recordings}

As previously reported in other polysomnographic studies, ${ }^{14,30}$ electroencephalographic (EEG) recordings and electrode placement were performed according to the $10-20$ system $^{31}$ and the polysomnographic montage included at least 
19 EEG channels (Fp2, Fp1, F3, F4, F7, F8, C3, C4, T3, T4, P3, P4, T5, T6, O1, O2, Fz, Cz, Pz) referenced to the contralateral mastoid, left and right electro-oculogram, chin electromyogram, left and right tibialis electromyogram, electrocardiogram (one derivation), nasal cannula, thorax and abdominal effort, peripheral oxygen saturation, pulse, and position sensors.

The recordings were carried out using a Brain Quick Micromed System 98 recording machine, and signals were sampled at $256 \mathrm{~Hz}$ and stored on a hard disk for further analysis. EEG signals were digitally band-pass filtered at 0.1-120 Hz, with 12-bit A/D precision. Sleep signals were sampled at $200 \mathrm{~Hz}$ or $256 \mathrm{~Hz}$ and stored on a hard disk in European data format ${ }^{32}$ for further analysis. EEG signals were first acquired with a wide band analog filter $(0.001-70 \mathrm{~Hz})$ and then digitally band-pass filtered at $0.1-50 \mathrm{~Hz}$. All recordings started at the subjects' usual bedtime and continued until spontaneous morning awakening.

\section{Sleep stage scoring}

Sleep was subdivided into 30 -second epochs, and sleep stages were scored using standard criteria. ${ }^{33}$ The following conventional sleep parameters were evaluated:

- Time in bed

- Sleep period time: time from sleep onset to end of sleep

- Total sleep time: time from sleep onset to the end of the final sleep epoch minus time awake

- Sleep latency: time from lights out to sleep onset, defined as the first of two consecutive epochs of stage 1 sleep or one epoch of any other stage, in minutes

- REM latency: time from sleep onset to the first REM sleep epoch

- Number of stage shifts/hour

- Number of awakenings/hour

- Sleep efficiency: percentage ratio between total sleep time and time in bed (total sleep time/time in bed * 100)

- Percentage of sleep period time spent in wakefulness after sleep onset, ie, time spent awake between sleep onset and end of sleep

- Percentage of sleep period time spent in sleep stages 1 (S1\%) and 2 (S2\%), slow-wave sleep (SWS\%), and REM sleep (REM\%).

All variables were analyzed by Hypnolab 1.2 sleep software analysis (SWS Soft, Troina, Italy). All recordings were visually scored by one of the investigators (MC), and the sleep parameters derived were tabulated for statistical analysis.
In order to exclude the presence of sleep-related breathing disorders, nocturnal respiratory parameters (ie, central, obstructive, and mixed apnea events) were counted using standard criteria. ${ }^{34}$ The apnea-hypopnea index was defined as the number of apneas and hypopneas per hour of total sleep time; an obstructive apnea index $>5$ was selected as the cutoff for normality. ${ }^{35,36}$

Standard criteria were used to identify episodes of periodic limb movements. The frequency of leg movements was represented as the periodic leg movement index (number/hour of total sleep time). Episodes of periodic limb movements were defined as leg movements with an amplitude increase of $8 \mu \mathrm{V}$ above the baseline value, a duration of $0.5-10$ seconds, a period length between two consecutive movements of 5-90 seconds, and a minimum of four consecutive movements. ${ }^{37}$ A periodic leg movement index $\geq 5$ was considered abnormal.

\section{Statistical analysis}

Comparisons between differences in sleep architecture at baseline and at the end of 6 months of treatment in insomniac subjects on Sea-Band therapy were done using the analysis of variance test. $P$ values $<0.05$ were considered to be statistically significant. The commercially available software Statistica version 6 (StatSoft Inc, Tulsa, OK) was used for all statistical tests. Statistical power was estimated using software for analysis available online (http://www.dssresearch. com/toolkit/spcalc/power.asp) for the polysomnographic findings at baseline and at the end of 6 months of treatment. The alpha error level of the confidence interval was $5 \%$.

\section{Results}

As shown in Table 1, at the end of 6 months of treatment, the study sample showed a significant increase in all macrostructural parameters of sleep duration (time in bed $526.680 \pm 65.642$ minutes, sleep period time 506.626 \pm 66.170 minutes, and total sleep time $437.957 \pm 68.344$ minutes) compared with baseline [time in bed $458.760 \pm 58.397$ minutes $(P=0.01)$, sleep period time $422.188 \pm 55.142$ minutes $(P<0.01)$, and total sleep time $336.352 \pm 59.893$ minutes $(P<0.001)]$. Moreover, after 6 months of acupressure therapy, insomniac subjects reveal a reduction in sleep onset latency $(36.572 \pm 13.217$ minutes versus $20.054 \pm 12.132$ minutes, $P<0.001$ ), in wake after sleep onset (sleep period time [spt] $20.645 \pm 6.719$ minutes versus $13.763 \pm 4.480$ minutes, $P<0.001)$ and in Stage $2(\mathrm{~S} 2)$ representation (S2-spt $32.331 \pm 6.119$ minutes versus $22.901 \pm 4.335$ minutes, $P<0.001$; S2-total sleep time [tst] $40.704 \pm 6.836$ minutes versus $26.500 \pm 4.572$ minutes, $P<0.001$ ). 
Table I Comparison of sleep macrostructural characteristics in insomniac subjects at baseline and after 6 months of treatment with the Sea-Bands ${ }^{\circledR}$ HT-7 system

\begin{tabular}{|c|c|c|c|}
\hline & $\begin{array}{l}\text { T0 } \\
n=25\end{array}$ & $\begin{array}{l}\text { TI } \\
n=25\end{array}$ & $P$ \\
\hline TIB-min & $458.760 \pm 58.397$ & $526.680 \pm 65.642$ & 0.010 \\
\hline SPT-min & $422.188 \pm 55.142$ & $506.626 \pm 66.170$ & $<0.01$ \\
\hline TST-min & $336.352 \pm 59.893$ & $437.957 \pm 68.344$ & $<0.001$ \\
\hline SOL-min & $36.572 \pm 13.217$ & $20.054 \pm 12.132$ & $<0.001$ \\
\hline FRL-min & $138.976 \pm 77.132$ & $125.078 \pm 69.419$ & NS \\
\hline SS-h & $12.924 \pm 2.568$ & $12.278 \pm 2.440$ & NS \\
\hline AWN-h & $6.828 \pm 2.048$ & $6.145 \pm 1.843$ & NS \\
\hline SE\% & $72.983 \pm 5.912$ & $82.930 \pm 5.060$ & $<0.001$ \\
\hline WASO-min & $85.836 \pm 26.948$ & $68.669 \pm 21.558$ & NS \\
\hline $\mathrm{SI}-\mathrm{min}$ & $14.360 \pm 10.384$ & $12.206 \pm 8.826$ & NS \\
\hline $\mathrm{S} 2-\mathrm{min}$ & $137.184 \pm 35.503$ & $116.606 \pm 30.177$ & NS \\
\hline SWS-min & $108.876 \pm 22.694$ & $225.619 \pm 28.986$ & $<0.001$ \\
\hline REM-min & $75.932 \pm 30.780$ & $83.525 \pm 33.858$ & NS \\
\hline WASO-spt & $20.645 \pm 6.719$ & $13.763 \pm 4.480$ & $<0.001$ \\
\hline $\mathrm{SI} \%$ & $3.479 \pm 2.684$ & $2.464 \pm 1.901$ & NS \\
\hline $\mathrm{S} 2 \%$ & $32.331 \pm 6.119$ & $22.901 \pm 4.335$ & $<0.001$ \\
\hline SWS\% & $25.944 \pm 5.477$ & $44.737 \pm 4.467$ & $<0.001$ \\
\hline REM\% & $|7.60| \pm 6.098$ & $16.134 \pm 5.590$ & NS \\
\hline SI-tst & $4.461 \pm 3.557$ & $2.884 \pm 2.272$ & NS \\
\hline S2-tst & $40.704 \pm 6.836$ & $26.500 \pm 4.572$ & $<0.001$ \\
\hline SWS-tst & $32.909 \pm 7.683$ & $52.067 \pm 6.497$ & $<0.001$ \\
\hline REM-tst & $21.926 \pm 6.852$ & $18.549 \pm 6.003$ & NS \\
\hline
\end{tabular}

Notes: An analysis of variance test was performed. $P$ values $<0.05$ were considered to be statistically significant.

Abbreviations: NS, not significant; min, minutes; TIB, time in bed; SPT, sleep period time; TST, total sleep time; SOL, sleep onset latency; FRL, first REM sleep latency; SS, stage shifts; AWN, awakenings; SE, sleep efficiency; WASO, wake time after sleep onset; SI, sleep stage I; S2, sleep stage 2; SWS, slow-wave sleep; REM, rapid eye movement sleep percentage; T0, baseline; TI, after 6 months of treatment.

Moreover, the study group show a significant increase in sleep efficiency $(\mathrm{SE}) \%(72.983 \pm 5.912$ versus $82.930 \pm 5.060$, $P<0.001$ ) and in SWS representation (SWS-min $108.876 \pm 22.694$ versus $225.619 \pm 28.986, P<0.001$; SWSspt $25.944 \pm 5.477$ versus $44.737 \pm 4.467, P<0.001$; and SWS-tst $32.909 \pm 7.683$ versus $52.067 \pm 6.497, P<0.001$, Table 1).

The statistical power calculated showed the following values: $100 \%$ for total sleep time-minutes, percent sleep efficiency, SWS-minutes, S2\%, SWS\%, S2-total sleep time, SWS-total sleep time, $99.9 \%$ for the sleep period timeminutes, $99.8 \%$ for the sleep onset latency-minutes, and $98.3 \%$ for the time in bed-minutes.

\section{Discussion}

Sleep is a vital physiological process with important restorative functions. Notable quantitative and qualitative changes in sleep occurring with age include more sleep fragmentation, earlier awakening, and reduced slow wave sleep. Age-related changes in the distribution of sleep cycles represent only a small piece of the complex array of chronobiological changes in physiological systems that accompany the aging process. Alternation between wakefulness and sleep, as well as the structure of sleep itself, are regulated by the interaction of outputs of the endogenous circadian pacemaker, ${ }^{38}$ located in the suprachiasmatic nucleus of the hypothalamus, ${ }^{39,40}$ and the homeostatic process. ${ }^{41,42}$

Moreover, the homeostatic process is thought to reflect the need for sleep which builds up during sustained wakefulness and dissipates during sleep. Slow wave sleep and slow wave activity, ie, EEG power density in the $0.75-4.5 \mathrm{~Hz}$ range, are considered to be markers of this process because they exhibit a predictive quantitative relationship with the duration of wakefulness and sleep. ${ }^{43}$ In this respect, the increasing time spent in slow wave sleep and the reducing sleep fragmentation (expressed by reduction in wake after sleep onset) in our sample could be interpreted as direct signs of improved sleep quality.

On the other hand, adolescents require more sleep than prepubertal youngsters, but frequently get less sleep than they need. Transition to an earlier school start time, along with sleep phase delay, significantly affects sleep quality, the sleep/wake schedule, and daytime behavior in teenagers. The combination of phase advance, late-night activities or jobs, and early morning school demands can significantly reduce the time available for sleep. In light of such considerations, the efficacy of HT-7 acupressure in improving the total sleep time in our sample could represent improvement in activation of the neuroendocrine system linked to sleep efficiency (ie, melatonin secretion) which is essential for cognition, ${ }^{14}$ mood regulation, ${ }^{3}$ and quality of life according to developmental age.

It is reasonable to say that complaints of insomnia may go unheeded either because of under-reporting to parents or lack of specialized prevention and early detection health programs, which seem to be of great importance and for which there is a pressing need. The confidentiality of our data collection enabled this need to emerge, and it would be important to promote a similar climate in any attempt to address this kind of problem in the future. Confidentiality is particularly important for adolescents, given that this age is, by definition, a time of fragility and vulnerability, making the adolescent reluctant to acknowledge any problems which could be perceived as faults and be unwilling to seek appropriate help. ${ }^{44}$ Therefore, acupressure could be considered as a noninvasive, safe, and effective method to treat insomnia with good compliance and no adverse effects. 
The strength of our findings lies in the use of gold standard polysomnography for assessment of sleep alterations in order to circumvent subjective reporting by patients. The main limitation of our study is the size of the study population and the subjects being recruited from the same urban area. Notwithstanding this limitation, our results suggest that acupressure could be a safety, effective, and cost-effective therapy for the management of psychophysiological insomnia in adolescence, even if future sources are needed.

\section{Acknowledgment}

The Sea-Band wristbands were kindly provided to the patients by Consulteam srl, Como, Italy.

\section{Disclosure}

The authors report no conflicts of interest in this work.

\section{References}

1. Stein MA, Mendelsohn J, Obermeyer WH, Amromin J, Benca R. Sleep and behavior problems in school-aged children. Pediatrics. 2001;107:E60.

2. Heussler H, Chan P, Price AM, Waters K, Davey MJ, Hiscock H. Pharmacological and non-pharmacological management of sleep disturbance in children: an Australian Paediatric Research Network survey. Sleep Med. 2012:pii:S1389-9457(12)00389-9.

3. Carotenuto M, Esposito M, Parisi L, et al. Depressive symptoms and childhood sleep apnea syndrome. Neuropsychiatr Dis Treat. 2012;8:369-373.

4. Carotenuto M, Guidetti V, Ruju F, Galli F, Tagliente FR, Pascotto A. Headache disorders as risk factors for sleep disturbances in school aged children. J Headache Pain. 2005;6:268-270.

5. Carotenuto M, Santoro N, Grandone A, et al. The insulin gene variable number of tandem repeats (INS VNTR) genotype and sleep disordered breathing in childhood obesity. J Endocrinol Invest. 2009;32: $752-755$

6. Gregory A, O'Conner T. Sleep problems in childhood: a longitudinal study of developmental change and association with behavioural problems. J Am Acad Child Adolesc Psychiatry. 2002;41:964-971.

7. Faruqui F, Khubchandani J, Price JH, Bolyard D, Reddy R. Sleep disorders in children: a national assessment of primary care pediatrician practices and perceptions. Pediatrics. 2011;128:539-546.

8. Doghramji K. The epidemiology and diagnosis of insomnia. Am J Manag Care. 2006;12:S214-S220.

9. Gozal D, Pope DW Jr. Snoring during early childhood and academic performance at ages thirteen to fourteen years. Pediatrics. 2001;107:1394-1399.

10. Corbo GM, Forastiere F, Agabiti N, et al. Snoring in 9- to 15-year-old children: risk factors and clinical relevance. Pediatrics. 2001;108: 1149-1154.

11. Tarasiuk A, Greenberg-Dotan S, Simon-Tuval T, et al. Elevated morbidity and health care use in children with obstructive sleep apnea syndrome. Am J Respir Crit Care Med. 2007;175:55-61.

12. Dahl RE. The impact of inadequate sleep on children's daytime cognitive function. Semin Pediatr Neurol. 1996;3:44-50.

13. Pilcher JJ, Huffcutt AJ. Effects of sleep deprivation on performance: a meta-analysis. Sleep. 1996;19:318-326.

14. Esposito M, Carotenuto M. Borderline intellectual functioning and sleep: the role of cyclic alternating pattern. Neurosci Lett. 2010;485: 89-93.
15. Johnson EO, Chilcoat HD, Breslau N. Sleep problems and anxiety/ depression in childhood. Psychiatry Res. 2000;94:93-102.

16. Patten CA, Choi WS, Gillin C, Pierce JP. Depressive symptoms and cigarette smoking predict development and persistence of sleep problems in US adolescents. Pediatrics. 2000;106:E23.

17. Johnson EO, Breslau N. Sleep problems and substance use in adolescence. Alcohol Drug Depend. 2001;64:1-7.

18. Liu X, Zhou H. Sleep duration, insomnia and behavioral problems among Chinese adolescents. Psychiatry Res. 2002;111:75-85.

19. Johnson EO, Roth T, Schultz L, Breslau N. Epidemiology of DSM-IV insomnia in adolescence: lifetime prevalence, chronicity, and an emergent gender difference. Pediatrics. 200;117:e247-e256.

20. Carotenuto M, Esposito M, Precenzano F, Castaldo L, Roccella M. Cosleeping in childhood migraine. Minerva Pediatr. 2011;63:105-109.

21. Yeung WF, Chung KF, Poon MM, et al. Acupressure, reflexology, and auricular acupressure for insomnia: a systematic review of randomized controlled trials. Sleep Med. 2012;13:971-984.

22. Cross JR. Acupressure: Clinical Applications in Musculoskeletal Conditions, 1st ed. Boston, MA: Butterworth-Heinemann Medical; 2000.

23. National Cancer Institute. Acupuncture. 2007. Available from: http://www.cancer.gov/cancertopics/pdq/cam/acupuncture/ HealthProfessional. Accessed December 18, 2008.

24. Spence DW, Kayumov L, Chen A, et al. Acupuncture increases nocturnal melatonin secretion and reduces insomnia and anxiety: a preliminary report. J Neuropsychiatry Clin Neurosci. 2004;16:19-28.

25. Yuksek MS, Erdem AF, Atalay C, Demirel A. Acupressure versus oxybutynin in the treatment of enuresis. $J$ Int Med Res. 2003;31:552-556.

26. Hsieh $\mathrm{CH}$. The effects of auricular acupressure on weight loss and serum lipid levels in overweight adolescents. Am J Chin Med. 2010;38:675-682.

27. Allais G, Rolando S, Castagnoli Gabellari I, et al. Acupressure in the control of migraine-associated nausea. Neurol Sci. 2012;33 Suppl 1: S207-S210.

28. Carotenuto M, Esposito M, Pascotto A. Facial patterns and primary nocturnal enuresis in children. Sleep Breath. 2011;15:221-227.

29. Agnew H, Webb W, Williams R. The first night effect: an EEG study of sleep. Psychophysiology. 1966;2:263-266.

30. Carotenuto M, Esposito M, D'Aniello A, et al. Polysomnographic findings in Rett syndrome: a case-control study. Sleep Breath. March 7, 2012.

31. Jasper HH. The 10-20 electrode system of the International Federation. Electroencephalogr Clin Neurophysiol. 1958;10:370-375.

32. Kemp B, Värri A, Rosa AC, Nielsen KD, Gade J. A simple format for exchange of digitized polygraphic recordings. Electroencephalogr Clin Neurophysiol. 1992;82:391-393.

33. Rechtschaffen A, Kales A. A Manual of Standardized Terminology, Techniques, and Scoring System for Sleep Stages of Human Subjects. Washington, DC: Public Health Service, US Government Printing Office; 1968.

34. American Thoracic Society. Standards and indications for cardiopulmonary sleep studies in children. Am J Respir Crit Care Med. 1996;153: 866-878.

35. Marcus CL, Omlin KJ, Basinki DJ, et al. Normal polysomnographic values for children and adolescents. Am Rev Respir Dis. 1992;146: $1235-1239$.

36. Traeger N, Schultz B, Pollock AN, Mason T, Marcus CL, Arens R. Polysomnographic values in children 2-9 years old: additional data and review of the literature. Pediatr Pulmonol. 2005;40:22-30.

37. Zucconi M, Ferri R, Allen R, et al. The official World Association of Sleep Medicine (WASM) standards for recording and scoring periodic leg movements in sleep (PLMS) and wakefulness (PLMW) developed in collaboration with a task force from the International Restless Legs Syndrome Study Group (IRLSSG). Sleep Med. 2006;7:175-183.

38. Czeisler CA, Weitzman ED, Moore-Ede MC, Zimmerman JC, Knauer RS. Human sleep: its duration and organization depend on its circadian phase. Science. 1980;210:1264-1267. 
39. Morin LP. Neuroanatomy of the extended circadian rhythm system. Exp Neurol. July 2, 2012. [Epub ahead of print.]

40. Farajnia S, Michel S, Deboer T, et al. Evidence for neuronal desynchrony in the aged suprachiasmatic nucleus clock. J Neurosci. 2012;32:5891-5899.

41. Vyazovskiy VV, Tobler I. The temporal structure of behaviour and sleep homeostasis. PLoS One. 2012;7:e50677.

42. Paech GM, Ferguson SA, Sargent C, Kennaway DJ, Roach GD. The relative contributions of the homeostatic and circadian processes to sleep regulation under conditions of severe sleep restriction. Sleep. 2012;35:941-948.
43. Jenni OG, Achermann P, Carskadon MA. Homeostatic sleep regulation in adolescents. Sleep. 2005;28:1446-1154.

44. Siomos KE, Avagianou PA, Floros GD, et al. Psychosocial correlates of insomnia in an adolescent population. Child Psychiatry Hum Dev. 2010;41:262-273

\section{Publish your work in this journal}

Neuropsychiatric Disease and Treatment is an international, peerreviewed journal of clinical therapeutics and pharmacology focusing on concise rapid reporting of clinical or pre-clinical studies on a range of neuropsychiatric and neurological disorders. This journal is indexed on PubMed Central, the 'PsycINFO' database and CAS.

The manuscript management system is completely online and includes a very quick and fair peer-review system, which is all easy to use. Visit http://www.dovepress.com/testimonials.php to read real quotes from published authors.

Submit your manuscript here: http://www.dovepress.com/neuropsychiatric-disease-and-treatment-journal 